Pesq. Vet. Bras. 35(4):562-568, junho 2015

DOI: 10.1590/S0100-2015000600013

\title{
Epidemiologia e distribuição de lesões extrarrenais de uremia em 161 cães $^{1}$
}

\author{
Isadora P. Silveira ${ }^{2}$, Maria Andréia Inkelmann ${ }^{3}$, Camila Tochetto $^{2}$, Fábio Brum Rosa ${ }^{2}$, \\ Rafael A. Fighera ${ }^{4}$, Luiz F. Irigoyen ${ }^{4}$ e Glaucia D. Kommers ${ }^{4 *}$
}

\begin{abstract}
Silveira I.P., Inkelmann M.A., Tochetto C., Rosa F.B., Fighera R.A, Irigoyen L.F., \& Kommers G.D. 2015. [Epidemiology and distribution of extrarenal lesions of uremia in 161 dogs.] Epidemiologia e distribuição de lesões extrarrenais de uremia em 161 cães. Pesquisa Veterinária Brasileira 35(6):562-568. Laboratório de Patologia Veterinária, Departamento de Patologia, Universidade Federal de Santa Maria, Camobi, Santa Maria, RS 97105-900, Brazil.E-mail: glaukommers@yahoo.com

The aim of this study was to determine the epidemiology and the morphological characteristics (including the anatomic localization) of the extrarenal uremic lesions, as well as to describe the main lesions of the urinary system associated with the occurrence of uremia, through analysis of the protocols of necropsies performed in dogs from January 1996 to December 2012 (17 years) at the Laboratório de Patologia Veterinária of the Universidade Federal de Santa Maria. A total of 4,201 dogs were necropsied and 161 (3.8\%) had extrarenal uremic lesions. In 134 dogs (83.2\%) clinical signs associated with uremia were reported. The extrarenal lesions more often observed, in descending order of prevalence, were ulcerative and hemorrhagic gastritis (56.5\%), soft-tissue mineralization (55.9\%), pulmonary edema (47.2\%), ulcerative stomatitis and/or glossitis (30.4\%), endocarditis/atrial and aortic thrombosis (28.6\%), parathyroid hyperplasia (9.3\%), fibrous osteodystrophy $(8.1 \%)$, anemia $(6.2 \%)$, ulcerative laryngitis $(5 \%)$, ulcerative and hemorrhagic enteritis $(3.7 \%)$, fibrinonecrotic esophagitis $(1.9 \%)$, and fibrinous pericarditis $(1.9 \%)$. In most of the cases, the extrarenal lesions of uremia were due to prolonged azotemia secondary to severe renal lesions, such as interstitial nephritis and glomerulonephritis (the most prevalent ones).
\end{abstract}

INDEX TERMS: Azotemia, diseases of dogs, extrarenal lesions, uremia.

RESUMO.- Com o objetivo de determinar a epidemiologia e as características morfológicas, incluindo a localização anatômica, das lesões extrarrenais de uremia, bem como determinar as principais lesões do sistema urinário associadas à ocorrência de uremia, foram revisados os proto-

\footnotetext{
${ }^{1}$ Recebido em 18 de setembro de 2014.

Aceito para publicação em 28 de março de 2015.

Parte da Dissertação de Mestrado do primeiro autor.

${ }^{2}$ Programa de Pós-Graduação em Medicina Veterinária, área de concentração em Patologia e Patologia Clínica Veterinária, Centro de Ciência Rurais (CCR), Universidade Federal de Santa Maria (UFSM), Av. Roraima 1000, Camobi, Santa Maria, RS 97105-900, Brasil.

${ }^{3}$ Departamento de Estudos Agrários (DEAg), Curso de Medicina Veterinária, Universidade Regional do Noroeste do Estado do Rio Grande do Sul (Unijuí), Rua do Comércio 3000, Bairro Universitário, Ijuí, RS 98700-000, Brasil.

${ }^{4}$ Laboratório de Patologia Veterinária, Departamento de Patologia, CCR-UFSM, Av. Roraima 1000, Camobi, Santa Maria, RS 97105-900. *Autor para correspondência: glaukommers@yahoo.com
}

colos de necropsias de cães realizadas no Laboratório de Patologia Veterinária da Universidade Federal de Santa Maria de janeiro de 1996 a dezembro de 2012 (17 anos). Nesse período foram necropsiados 4.201 cães, sendo que $161(3,8 \%)$ apresentaram lesões extrarrenais de uremia. Em 134 cães $(83,2 \%)$ foram descritos sinais clínicos associados à uremia. As lesões extrarrenais mais frequentes, em ordem decrescente, foram: gastrite ulcerativa e hemorrágica $(56,5 \%)$, mineralização de tecidos moles $(55,9 \%)$, edema pulmonar $(47,2 \%)$, estomatite e/ou glossite ulcerativa $(30,4 \%)$, endocardite/trombose atrial e aórtica $(28,6 \%)$, hiperplasia das paratireoides $(9,3 \%)$, osteodistrofia fibrosa $(8,1 \%)$, anemia $(6,2 \%)$, laringite ulcerativa $(5 \%)$, enterite ulcerativa/hemorrágica $(3,7 \%)$, esofagite fibrinonecrótica $(1,9 \%)$ e pericardite fibrinosa (1.9\%). Na maioria dos casos as lesões extrarrenais de uremia foram decorrentes de azotemia prolongada por lesões renais graves, sendo as mais prevalentes a nefrite intersticial e a glomerulonefrite. 
TERMOS DE INDEXAÇÃO: Azotemia, doenças de cães, lesões extrarrenais, uremia.

\section{INTRODUÇÃO}

Os rins têm como funções básicas a excreção de metabólitos tóxicos ao organismo através da depuração sanguínea, reabsorção de fluidos e alguns processos endócrinos, como a produção de eritropoietina (Confer \& Panciera 1998). Quando a função renal está diminuída, com aproximadamente $75 \%$ dos néfrons sem funcionar adequadamente (Maxie \& Newman 2007), ocorre o aumento intravascular de ureia e creatinina, condição denominada azotemia (Newman et al. 2009). Entretanto, a azotemia também pode ocorrer por causas pré-renais ou pós-renais. Uma condição que reduza o fluxo sanguíneo para os rins pode resultar em azotemia pré-renal, como nos casos de hipovolemia, hipotensão e formação de trombo em aorta ou arterial renal. A azotemia pós-renal geralmente é causada por obstrução do fluxo urinário ou ruptura no trato urinário inferior (Grauer 2010).

A uremia é uma síndrome resultante de azotemia prolongada (Cheville 1979), quando ocorre a retenção de compostos que, em condições normais, seriam metabolizados e excretados pelos rins. Esses compostos são bioquimicamente ativos e são chamados de toxinas urêmicas. 0 acúmulo dessas toxinas tem um impacto negativo sobre diversas funções corporais e resultam em uma gradual intoxicação endógena (Vanholder et al. 2008). Consequentemente, cães urêmicos têm sinais clínicos e lesões multissistêmicas (extrarrenais) devido à insuficiência renal (Newman et al. 2009). A ocorrência de lesões extrarrenais de uremia, identificadas clinicamente ou durante a necropsia, depende do tempo de sobrevida do animal no estado urêmico. Desta forma, a gravidade dessas lesões é maior nos casos de insuficiência renal crônica (Newman et al. 2009).

Os cães são a espécie mais prevalente nas necropsias realizadas no Laboratório de Patologia Veterinária da Universidade Federal de Santa Maria (LPV-UFSM). Considerando-se que, no período de 1999 a 2010, aproximadamente $33 \%$ dos cães necropsiados no LPV-UFSM apresentaram lesões no sistema urinário e que a uremia foi observada em um número expressivo de casos (Inkelmann et al. 2012), os principais objetivos deste estudo retrospectivo foram determinar a epidemiologia e as características morfológicas, incluindo a localização das lesões extrarrenais de uremia, bem como determinar as principais lesões do sistema urinário associadas à ocorrência de uremia.

\section{MATERIAL E MÉTODOS}

Os protocolos de necropsias de cães realizadas no LPV-UFSM de janeiro de 1996 a dezembro de 2012 (17 anos) foram revisados. 0 critério utilizado para a seleção dos casos foi a ocorrência de lesões extrarrenais de uremia. Foram computados todos os cães necropsiados no período e o total de cães com lesões extrarrenais de uremia. Dos protocolos de necropsias foram obtidos dados referentes ao histórico clínico, à epidemiologia (sexo, raça, idade) e ao tipo de lesão primária. Em relação à faixa etária, os cães foram classificados como filhotes (menos de um ano de idade), adultos (1-9 anos de idade) ou idosos (10 anos de idade ou mais), con- forme previamente realizado por Fighera et al. (2008). Quanto à raça, os cães foram divididos em com raça definida (CRD) ou sem raça definida (SRD).

As lesões extrarrenais foram analisadas e agrupadas de acordo com sua localização e com suas características morfológicas (macroscópicas e microscópicas), de acordo com o proposto na literatura (Newman et al. 2009). Também foram computadas as lesões primárias do sistema urinário que resultaram em uremia.

\section{RESULTADOS}

No período de janeiro de 1996 a dezembro de 2012 foram necropsiados 4.201 cães no LPV-UFSM. Desses, 161 (3,8\%) apresentaram lesões extrarrenais de uremia. Cães que tinham raça definida foram a maioria, totalizando 95 casos (59\%); as raças mais frequentemente afetadas foram Dálmata $(11 / 95 ; 11,6 \%)$ e Poodle $(11 / 95 ; 11,6 \%)$. Dez eram filhotes $(6,2 \%), 85(52,8 \%)$ eram adultos e $61(37,9 \%)$ eram cães idosos; em cinco casos $(3,1 \%)$ a idade não foi informada nos protocolos. Dos 161 casos, 90 (55,9\%) cães eram machos e $71(44,1 \%)$ eram fêmeas.

Em 134 cães $(83,2 \%)$ foram descritos sinais clínicos associados à uremia. 0 sinal clínico mais frequente foi vômito (86/134; 64,2\%), seguido de anorexia (64/134; 47,8\%), apatia $(37 / 134 ; 27,6 \%)$ e diarreia $(28 / 134 ; 20,9 \%)$. Menos frequentemente foram relatados emagrecimento, úlceras na cavidade oral e na língua. Apenas em um caso relatou-se hálito urêmico. Em 55 casos havia informações sobre ocorrência de azotemia (elevação dos índices de ureia e creatinina). Os valores da ureia variaram entre 80 e $738 \mathrm{mg} / \mathrm{dL}$, enquanto que os de creatinina variaram de 2,8 a $35,7 \mathrm{mg} /$ dL. Porém, destes 55 casos, em 11 havia informação de que esses índices estavam aumentados, sem informar o valor.

Quanto à origem da azotemia prolongada que levou à uremia, a maioria dos casos foi de origem renal, perfazendo um total de 139 cães $(86,3 \%)$. As causas mais comuns de lesão renal que resultaram em uremia foram, em ordem decrescente, a combinação de duas ou mais lesões renais (53/139; 38\%), nefrite intersticial $(25 / 139 ; 18 \%)$, glomerulonefrite $(20 / 139 ; 14 \%)$, fibrose $(12 / 139 ; 8,6 \%)$, necrose tubular $(10 / 139 ; 7,2 \%)$, degeneração tubular $(4 / 139 ; 2,9 \%)$, pielonefrite $(3 / 139 ; 2,2 \%)$, glomeruloesclerose $(2 / 139 ; 1,4 \%)$, hidronefrose $(2 / 139 ; 1,4 \%)$, infarto $(2 / 139 ; 1,4 \%)$, nefrolitíase $(2 / 139,1,4 \%)$, amiloidose (1/139; 0,7\%), carcinoma (1/139; 0,7\%), necrose de crista renal $(1 / 139 ; 0,7 \%)$ e pionefrose $(1 / 139 ; 0,7 \%)$. As lesões renais diagnosticadas, em 105 (75,5\%) cães resultaram em insuficiência renal crônica (IRC), enquanto que, em 34 $(24,5 \%)$ cães, resultaram em insuficiência renal aguda. Em 22 casos $(13,7 \%)$ a azotemia prolongada foi de origem pós-renal, dos quais 20 (91\%) foram associados à obstrução uretral e dois (9\%) à obstrução uretral com ruptura da uretra. Casos de uremia associada à azotemia pré-renal não foram diagnosticados no período estudado.

0 tipo e a frequência de aparecimento das lesões extrarrenais (macroscópicas e/ou microscópicas) de uremia nos 161 cães encontram-se no Quadro 1 e serão detalhados a seguir, em ordem decrescente de prevalência. Não foi observado um padrão para a ocorrência das lesões que, na maioria das vezes, eram multissistêmicas. 
Quadro 1. Tipo e frequência de aparecimento de lesões extrarrenais de uremia em 161 cães

\begin{tabular}{lcc}
\hline Lesão & $\begin{array}{c}\text { Número de } \\
\text { ocorrências/cães }\end{array}$ & $\begin{array}{c}\text { \% sobre o total } \\
\text { de cães* }\end{array}$ \\
\hline Gastrite ulcerativa e hemorrágica & $135 / 91$ & 56,50 \\
com ulceração e hemorragia & 91 & - \\
com odor amoniacal & 31 & - \\
com vasculite e trombose & 13 & - \\
Mineralização de tecidos moles & $189 / 90$ & 55,90 \\
pulmões & 40 & - \\
coração & 37 & - \\
pleura e músculos intercostais & 36 & - \\
estômago & 34 & - \\
rins & 27 & - \\
aorta & 5 & - \\
laringe & 5 & - \\
diafragma & 1 & - \\
intestino delgado & 1 & - \\
língua & 1 & - \\
traqueia & 1 & - \\
Edema pulmonar & $76 / 76$ & 47,20 \\
Estomatite e/ou glossite ulcerativa & $52 / 49$ & 30,40 \\
com úlceras multifocais & 49 & - \\
com trombose & 3 & - \\
Endocardite/trombose atrial e aórtica & $46 / 46$ & 28,60 \\
Hiperplasia da paratireoide & $15 / 15$ & 9,30 \\
Osteodistrofia fibrosa & $13 / 13$ & 8,10 \\
Anemia & $10 / 10$ & 6,20 \\
Laringite ulcerativa & $8 / 8$ & 5,00 \\
Enterite ulcerativa/hemorrágica & $7 / 6$ & 3,70 \\
com ulceração e hemorragia & 6 & - \\
com trombose & 1 & - \\
Esofagite fibrinonecrótica & $3 / 3$ & 1,90 \\
Pericardite fibrinosa & $3 / 3$ & 1,90 \\
\hline Coniderando a ocorrenca & & \\
\hline
\end{tabular}

* Considerando a ocorrência simultânea de várias lesões extrarrenais de uremia num mesmo cão.

A lesão extrarrenal de uremia mais prevalente foi a gastrite ulcerativa e hemorrágica (Fig.1), observada em 91 casos (56,5\%), e caracterizada por erosões, úlceras, edema e avermelhamento da mucosa gástrica. 0 conteúdo gástrico era hemorrágico na grande maioria dos casos. Em 31 desses casos (34\%) a mucosa do estômago exalava forte odor amoniacal. Alterações vasculares como necrose fibrinoide (Fig.2), vasculite e trombose, foram descritas em 13 desses casos $(14,3 \%)$.

A segunda lesão mais prevalente, com um número quase equivalente de cães afetados que a primeira, foi a mineralização de tecidos moles, envolvendo múltiplos órgãos/ tecidos, que ocorreu em 90 cães (55,9\%). A mineralização ocorreu em 13 localizações diferentes, as quais foram, em ordem decrescente de prevalência: pulmões, coração, pleura/músculos intercostais, estômago, rins, aorta, laringe, diafragma, intestino delgado, língua e traqueia.

Os pulmões foram os órgãos onde a mineralização foi mais frequentemente observada, totalizando 40 casos. Os pulmões encontravam-se não colapsados, pálidos ou avermelhados, mais firmes e pesados (Fig.3). A mineralização nesses casos estava presente principalmente nos septos interalveolares (Fig.4), mas também foi observada na parede de arteríolas. Em 37 casos foi observada a mineralização no coração; no endocárdio (Fig.5 e 6) e/ou miocárdio (nas cardiomiofibras e vasos sanguíneos). Em 36 casos foi ob- servada mineralização da pleura parietal e músculos intercostais subjacentes, descrita como estriações brancas e elevadas, que acompanhavam o sentido das fibras musculares (Fig.7). Em 24 casos foi observada mineralização principalmente da porção média da mucosa do estômago; em quatro casos, somente a parede dos vasos sanguíneos da submucosa estava mineralizada e seis cães apresentavam mineralização, tanto da mucosa, como dos vasos da submucosa gástrica. Nos rins, os locais mais comuns de mineralização foram os túbulos renais e a cápsula de Bowman, totalizando 27 casos. A artéria aorta estava mineralizada em cinco casos; apresentava parede espessa e dura ao corte, por vezes, com áreas multifocais elevadas e brancas internamente. Também em cinco casos foram descritas placas brancas multifocais ou coalescentes na mucosa da laringe (Fig.8). Mineralização de diafragma, intestino delgado, língua e traqueia, foram descritas somente uma vez cada.

Edema pulmonar ocorreu em 76 cães $(47,2 \%)$. Nesses casos, os pulmões encontravam-se difusamente não colapsados e úmidos, fluindo líquido na superfície de corte. Em vários deles, histologicamente havia também depósitos alveolares de fibrina e infiltrado inflamatório principalmente de macrófagos e menor quantidade de neutrófilos (como parte da condição também conhecida como pneumopatia urêmica).

Estomatite e/ou glossite ulcerativas estavam presentes em 49 cães $(30,4 \%)$. Oito cães apresentaram as duas lesões simultaneamente, sete cães apresentaram somente estomatite e 34, somente glossite. Ambas as lesões foram identificadas pela presença de úlceras na mucosa oral (Fig.9) e na língua, principalmente nos bordos ventrais da língua, muitas vezes recobertas por uma película de fibrina. Dois cães tiveram infarto da ponta da língua (Fig.10).

Endocardite (mural) e trombose atrial e aórtica foram observadas em 46 casos $(28,6 \%)$. 0 local mais acometido $(31 / 46 ; 67,4 \%)$ foi o átrio esquerdo (Fig. 5), seguido do átrio direito $(6 / 46 ; 13 \%)$, do ventrículo esquerdo $(5 / 46$; $10,9 \%)$ e do ventrículo direito $(1 / 46 ; 2,2 \%)$. Em três deles não estava especificado o local da lesão. As lesões eram áreas elevadas, irregulares e brancacentas no endocárdio que, por vezes, estavam associadas a trombos. Nesses casos, além de mineralização frequente, havia necrose e infiltrado inflamatório misto. Somente um cão apresentou trombose aórtica, descrita como áreas esbranquiçadas e salientes aderidas à íntima da aorta, na base do coração.

Em 15 casos (9,3\%) foi descrito aumento bilateral das paratireoides e caracterizado como hiperplasia dessas glândulas (Fig.11). Osteodistrofia fibrosa foi descrita em 13 cães $(8,1 \%)$. Nesses casos, os ossos eram maleáveis e não ofereciam resistência à pressão. As costelas, quando tracionadas, fraturavam com facilidade.

A prevalência de anemia baseou-se nas informações clínicas (hematócrito) somadas aos achados de necropsia (mucosas pálidas, sangue aquoso e hipoplasia eritroide da medula óssea), perfazendo 10 casos (6,2\%). Em oito casos (5\%), observou-se laringite ulcerativa. Havia placas rugosas, elevadas, branco-amareladas, na mucosa da laringe.

As lesões de enterite foram classificadas como ulcerativas e hemorrágicas e totalizaram seis casos $(3,7 \%)$, sen- 

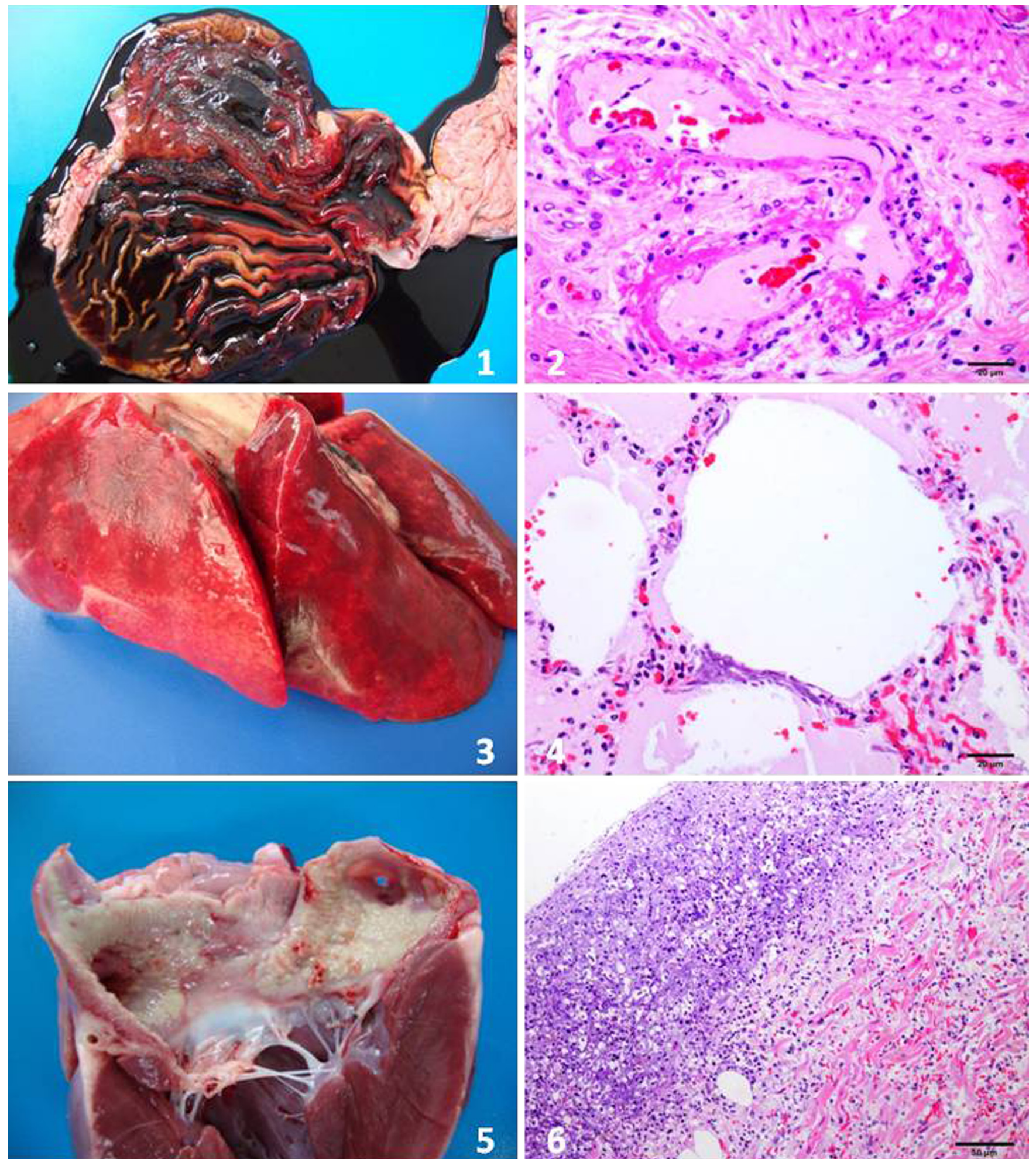

Fig.1. A mucosa do estômago está avermelhada, com conteúdo sanguinolento, na uremia de um cão.

Fig.3. Os pulmões estão armados, avermelhados com mineralização, na uremia de um cão.

Fig.5. Àreas elevadas, irregulares e brancacentas no endocárdio (endocardite associada à mineralização) do átrio esquerdo de um cão com uremia.

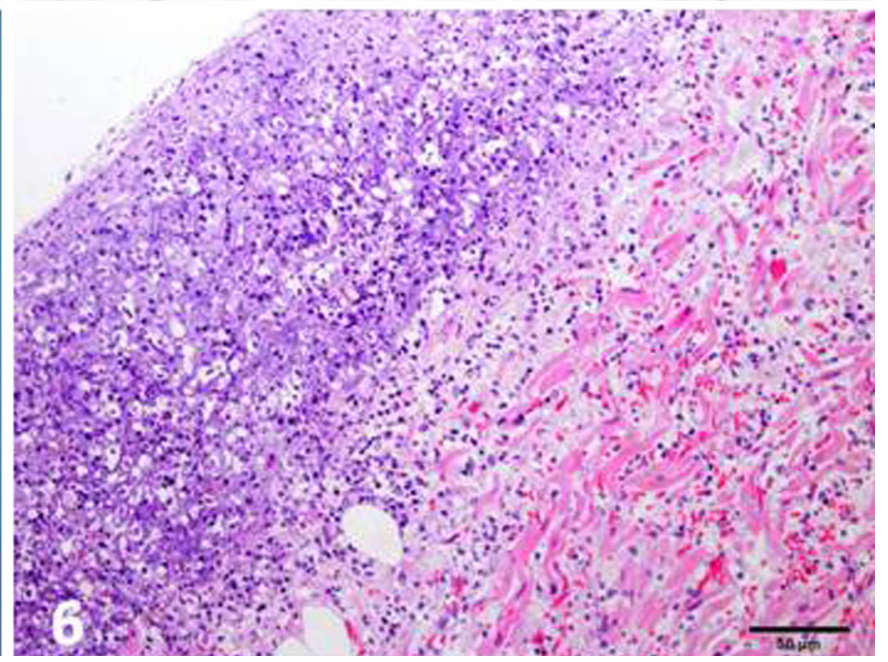

Fig.2. Necrose fibrinoide na parede da arteríola da submucosa no estômago de um cão com uremia. HE, obj.40x.

Fig.4. Deposição de material basofílico granular nos septos interalveolares (mineralização) no pulmão de um cão com uremia. Alguns alvéolos estão repletos de material eosinofílico amorfo (edema). HE, obj.40x.

Fig.6. Infiltrado inflamatório composto por grande quantidade de neutrófilos com deposição de fibrina e material basofílico granular (mineralização) no endocárdio atrial de um cão com uremia. HE, obj.20x. 

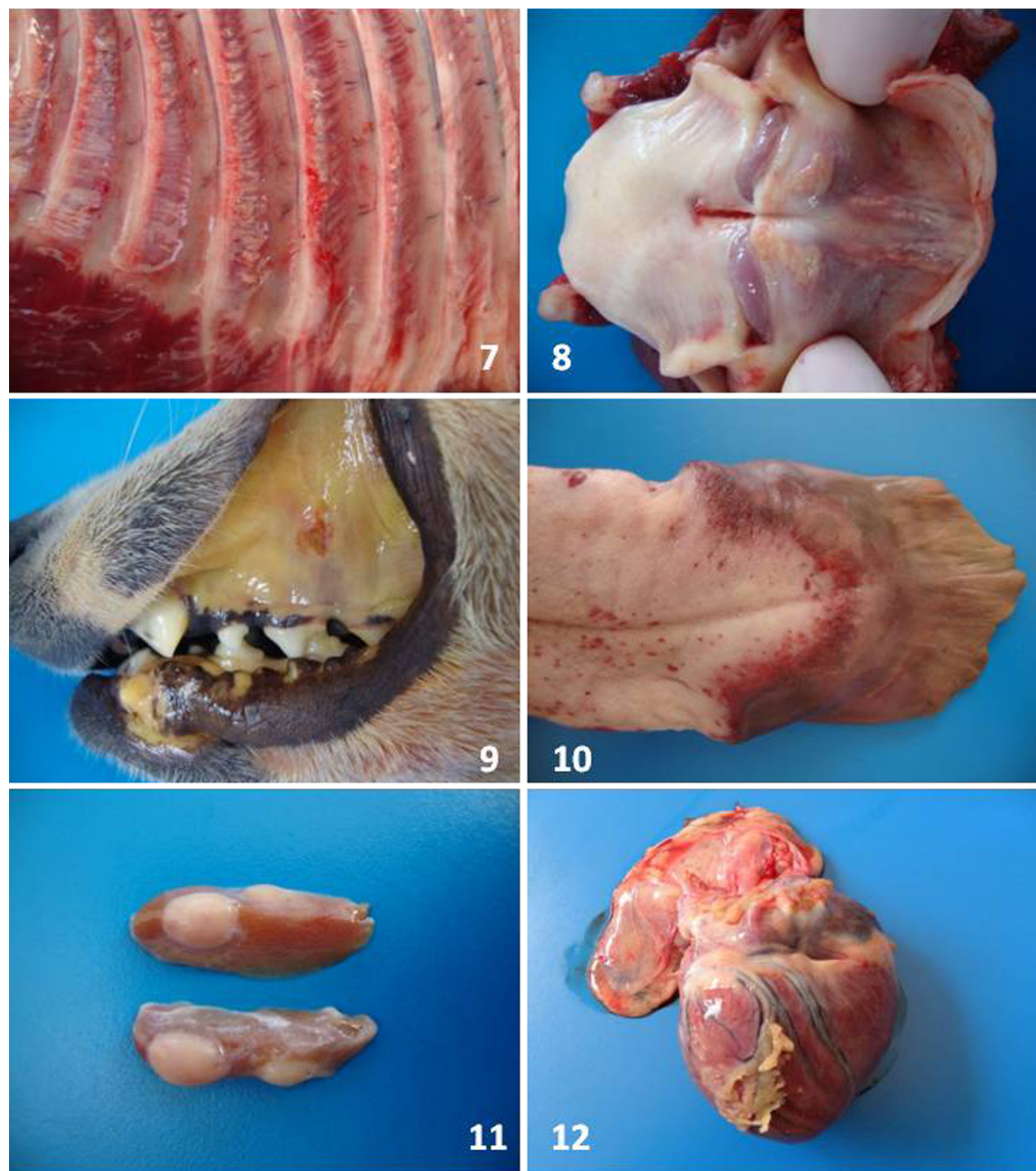

Fig.7. Mineralização da pleura parietal e músculos intercostais subjacentes, com estriações brancas e elevadas, que acompanham o sentido das fibras musculares, em um cão com uremia.

Fig.9. Ulceração focal na cavidade oral de um cão com uremia. A icterícia observada na mucosa deve-se à causa primária da insuficiência renal (leptospirose).

Fig.11. As paratireoides estão aumentadas de tamanho (hiperplasia) em um cão com uremia.

do que um deles apresentava odor amoniacal no intestino. Nesses casos, foram observadas úlceras e erosões, bem como petéquias e hiperemia na mucosa. Por vezes, o conteúdo intestinal estava enegrecido. Em um caso foi observada trombose nos vasos da lâmina própria.

Esofagite e pericardite foram observadas apenas em

Fig.8. Mineralização da laringe caracterizada por placas brancas multifocais na mucosa de um cão com uremia.

Fig.10. Área extensa de necrose da ponta da língua de um cão com uremia.

Fig.12. Fibrina aderida ao pericárdio visceral (pericardite fibrinosa) em um cão com uremia.

três cães $(1,9 \%)$ cada. As lesões de esofagite foram classificadas como fibrinonecróticas. Havia úlceras na mucosa esofágica, recobertas por fibrina e hiperemia difusa. A pericardite fibrinosa era caracterizada pela deposição de material fibrilar amarelado (fibrina) no pericárdio visceral (Fig.12). 


\section{DISCUSSÃO}

A prevalência de uremia nos cães necropsiados no LPV-UFSM foi de 3,83\%. Entretanto, quando analisados os cães com lesão no sistema urinário, essa prevalência é bem mais expressiva na rotina do laboratório (Inkelmann et al. 2012). As lesões extrarrenais de uremia ocorrem especialmente em cães com insuficiência renal crônica (IRC) (Maxie \& Newman, 2007), afetando principalmente cães de meia idade ou idosos (Polzin et al. 1992), o que pode ser confirmado neste estudo. Não há predisposição de raça para a ocorrência de IRC (Polzin et al. 1992). Embora as raças Dálmata e Poodle tenham sido mais prevalentes entre os cães estudados, não se pode atribuir uma maior suscetibilidade a elas, pois não se conhece a constituição racial da população de cães na região.

Os sinais clínicos frequentes de uremia relacionados ao sistema digestório, como anorexia e emagrecimento, são inespecíficos e podem anteceder outros sinais (Galvão et al. 2010). Anorexia e emagrecimento, juntamente com vômito, apatia e diarreia, foram relatados com grande frequência neste estudo, assim como relatado por outros autores (Polzin \& Osborne 1995, Dantas \& Kommers 1997). Esse fato está relacionado à alta prevalência de lesões encontradas no sistema digestório dos animais urêmicos, principalmente no estômago. A estimulação da zona deflagradora dos quimiorreceptores pelas toxinas urêmicas, a menor excreção renal de gastrina, a maior secreção de ácido gástrico e a irritação gastrointestinal secundária à uremia parecem contribuir para a ocorrência de vômitos e anorexia nessa síndrome (Grauer 2010). 0 hálito urêmico, informado somente em um dos históricos clínicos (mas que provavelmente ocorreu em mais casos), e o odor amoniacal exalado da mucosa estomacal, presente em 31 casos, possivelmente originam-se da degradação da ureia em amônia por ação da urease bacteriana (Polzin \& Osborne 1995).

Em qualquer situação que leve à azotemia prolongada (pré-renal, renal ou pós-renal), a excreção de metabólitos fica comprometida e pode haver o desenvolvimento de sinais clínicos e lesões extrarrenais de uremia (Cowgill \& Elliot 2008, Polzin et al. 2008). A presença de lesões em outros órgãos é descrita como mais frequente em animais com lesões renais do que por causas pré-renais ou pós-renais (Chew \& Dibartola 1992, Maxie \& Newman 2007). Isso foi confirmado nos resultados deste estudo que demonstraram que, na maioria das vezes, as lesões extrarrenais de uremia foram decorrentes de azotemia prolongada por lesões graves dos rins (azotemia renal), com poucos casos em que a azotemia foi decorrente da obstrução do trato urinário inferior (azotemia pós-renal). Casos de uremia associada à azotemia pré-renal não foram observados neste estudo provavelmente porque as causas desse mecanismo frequentemente levam à morte do animal antes do aparecimento de lesões extrarrenais visíveis.

A maioria das lesões que culminam em uremia são lesões renais e, dentre elas, a fibrose difusa, a glomerulonefrite e a nefrite túbulo-intersticial são as principais (Inkelmann et al. 2012). Neste estudo, essas lesões estão entre as lesões mais prevalentes que resultaram em uremia, assim como a associação de duas ou mais lesões renais.

A lesão mais prevalente neste estudo foi a gastrite ulcerativa e hemorrágica e sua patogênese parece estar asso- ciada à necrose vascular e à produção de amônia a partir da ureia por bactérias (Maxie \& Newman 2007). A amônia exerce um efeito cáustico sobre a mucosa (Barker et al. 2007). Dos cães que apresentaram gastrite, $13 \%$ tinham lesões vasculares (vasculite e trombose) descritas nos protocolos de necropsias. Em um estudo com 28 cães urêmicos, $54 \%$ apresentaram lesões vasculares gástricas (Peters et al. 2005). Focos de necrose e ulceração podem ser resultado da anóxia secundária à isquemia associada às lesões vasculares. Embora essa teoria seja aceita, trombose nem sempre foi encontrada em áreas de ulceração gástrica neste estudo, assim como descrito na literatura (Cheville 1979).

A mineralização de tecidos moles, envolvendo múltiplos órgãos e tecidos, está associada a alterações do metabolismo de cálcio e fósforo (Newman et al. 2009). 0 desequilíbrio do metabolismo de cálcio e de fósforo ocorre como consequência da perda gradativa da capacidade funcional dos rins (Lazaretti et al. 2006). A maioria dos animais apresenta hiperfosfatemia e níveis de cálcio baixos, o que promove estímulo das paratireoides e aumento da secreção de paratormônio (PTH), na tentativa de manutenção da homeostase do cálcio (Lazaretti et al. 2006). Desta forma ocorre mobilização de cálcio a partir do tecido ósseo (Newman et al. 2009). Com o tempo, esses eventos levam à hiperplasia das paratireoides, osteodistrofia fibrosa (substituição de tecido ósseo por tecido conjuntivo fibroso) e calcificação de tecidos moles (Newman et al. 2009).

Segundo a literatura, a deposição de cálcio em trato gastrointestinal, pleura, pulmões, miocárdio, endocárdio e rins é frequente em cães urêmicos, predominando a mineralização subpleural nos espaços intercostais (Maxie \& Newman 2007). No entanto, neste estudo, o local em que mais se observou mineralização foi nos pulmões, totalizando 40 casos. Já as outras lesões associadas ao desequilíbrio do metabolismo do cálcio e do fósforo, a hiperplasia de paratireoides e a osteodistrofia fibrosa, citadas anteriormente, foram pouco frequentes. Sabe-se que as lesões ósseas são menos comuns quando comparadas às lesões em outros sistemas (Thompson 2007). Vale salientar que os ossos muitas vezes não são histologicamente examinados durante os procedimentos de rotina, o que dificulta o diagnóstico da osteodistrofia fibrosa de origem renal em fases iniciais de desenvolvimento.

0 aumento da permeabilidade vascular é responsável por duas lesões extrarrenais de uremia: o edema pulmonar e a pericardite fibrinosa (Newman et al. 2009). Neste estudo, 76 cães apresentaram edema pulmonar. A formação de líquido rico em proteínas nos pulmões de pacientes urêmicos comprova o envolvimento deste mecanismo na patogênese do edema pulmonar (Rackow et al. 1978). Pericardite fibrinosa estava presente em somente três dos casos estudados. Em um estudo prévio realizado no LPV-UFSM, avaliando 72 cães com uremia no período de 1986 a 1995 (anterior ao período deste estudo), nenhum cão apresentou essa lesão no coração (Dantas \& Kommers 1997). A pericardite fibrinosa é uma complicação bem conhecida em pacientes humanos urêmicos, já em cães essa lesão é considerada rara (Chew \& Dibartola 1992).

Os mecanismos envolvidos em estomatite, glossite, enterite e esofagite ulcerativas, observadas em um menor número de casos neste estudo, são os mesmos citados anteriormente para as lesões gástricas (Newman et al. 2009). 
Lesões orais estão entre as complicações do sistema digestório mais importantes da uremia em cães. A necrose da ponta da língua, que é uma lesão observada ocasionalmente (Chew \& Dibartola 1992), possivelmente causada por trombose de vasos de maior calibre da língua com consequente isquemia, foi observada em dois cães deste estudo. As lesões intestinais assemelham-se às do estômago, mas são descritas como menos frequentes em cães, menos severas e sem mineralização (Newman et al. 2009). Porém, neste estudo observou-se a ocorrência de um caso de mineralização leve no intestino delgado.

A endocardite mural e a trombose atrial e aórtica podem ser atribuídas à degeneração ou necrose endotelial (Confer \& Panciera 1998). Essas lesões foram descritas em 46 cães deste estudo. A forma mais comum de endocardite mural em cães é a que ocorre na insuficiência renal (Maxie \& Robinson 2007). No cão urêmico, pode ocorrer degeneração focal subendotelial no endocárdio e, menos frequentemente, na superfície endotelial da aorta e do tronco pulmonar. Devido à predisposição à trombose por perda de anti-trombina III na urina, em cães com lesões glomerulares grandes trombos podem desenvolver-se nesses locais (Confer \& Panciera 1998). Neste estudo, somente um cão apresentou trombose aórtica.

A anemia associada à insuficiência renal é, em grande parte, devida à inadequada produção de eritropoietina (MacDougall 2001), mas o aumento da hemólise e a perda sanguínea também podem contribuir (Polzin et al. 1992). Além disso, em humanos, sabe-se que a vida das hemácias em pacientes urêmicos fica reduzida a aproximadamente a metade do observado em indivíduos sadios (Chew \& Dibartola 1992). Sugere-se também que outras substâncias possam desempenhar um papel na inibição da eritropoiese, incluindo as poliaminas, paratormônio (PTH) e algumas citocinas inflamatórias (MacDougall 2001). Neste estudo somente em 10 cães relatou-se a ocorrência de anemia. Porém, este número pode estar subestimado, pois a análise da medula óssea nem sempre é realizada na rotina.

\section{CONCLUSÕES}

Esse estudo, abordando epidemiologia e distribuição das lesões extrarrenais de uremia em caninos por um período de 17 anos, permitiu as seguintes conclusões:

Cães de meia idade e idosos foram mais propensos a desenvolver IRC e, consequentemente, uremia;

Os sinais clínicos mais diagnosticados em cães com uremia, em ordem decrescente de prevalência foram: vômito, anorexia, apatia, diarreia, úlceras em cavidade oral e língua e hálito urêmico;

Em $86,3 \%$ dos casos as lesões extrarrenais de uremia foram decorrentes de azotemia prolongada por lesões graves dos rins, sendo as mais comuns a nefrite intersticial e a glomerulonefrite;

As lesões extrarrenais de uremia mais frequentes, em ordem decrescente foram: gastrite ulcerativa e hemorrágica, mineralização de tecidos moles, edema pulmonar, estomatite e glossite ulcerativas, endocardite/trombose atrial e aórtica, hiperplasia de paratireoides, osteodistrofia fibrosa, anemia, laringite, enterite ulcerativa/hemorrágica, esofagite fibrinonecrótica e pericardite fibrinosa.

\section{REFERÊNCIAS}

Barker I.K., Van Dreumel A.A. \& Palmer N. 2007. The alimentary system, p.1-318. In: Maxie M.G. (Ed.), Jubb, Kennedy, and Palmer's Pathology of Domestic Animals. Vol.2. $5^{\text {th }}$ ed. Saunders Elsevier, St Louis.

Chew D.J. \& Dibartola S.P. 1992. Diagnóstico e fisiopatologia da moléstia renal, p.1975-2046. In: Ettinger S.J. (Ed.), Tratado de Medicina Interna Veterinária. Vol.4. 3aㅡ ed. Manole, São Paulo.

Cheville N.F. 1979. Uremic gastropathy in the dog. Vet. Pathol. 16:292-309.

Confer A.W. \& Panciera R.J. 1998. Sistema urinário, p.228-265. In: Carlton W.W. \& McGavin M.D. (Eds), Patologia Veterinária Especial de Thomson. $2^{\text {a }}$ ed. Artmed, São Paulo.

Cowgill L.D. \& Elliott D.A. 2008. Insuficiência renal aguda, p.1701-1721. In: Ettinger S.J. \& Feldman E.C. (Eds), Tratado de Medicina Interna Veterinária: doenças do cão e do gato. Vol.2. 5a ed. Guanabara Koogan, Rio de Janeiro.

Dantas A.F.M. \& Kommers G.D. 1997. Lesões extra-renais de uremia em 72 cães. Ciência Rural 27(2):301-306.

Fighera R.A., Souza T.M., Silva M.C., Brum J.S., Graça D.L., Kommers G.D., Irigoyen L.F. \& Barros C.S.L. 2008. Causas de morte e razões para eutanásia de cães da Mesorregião do Centro Ocidental Rio-Grandense (19652004). Pesq. Vet. Bras. 28(4):223-230.

Galvão A.L.B., Borges J.C., Vieira M.C., Ferreira G., Lega E. \& Pinto M. 2010. Alterações clinicas e laboratoriais de cães e gatos com doença renal crônica: revisão de literatura. Nucleus Animalium 2(1):23-40.

Grauer G.F. 2010. Distúrbios do trato urinário, p.609-696. In: Nelson R.W. \& Couto C.G. (Eds), Medicina Interna de Pequenos Animais. 4aa ed. Elsevier, Rio de Janeiro.

Inkelmann M.A., Kommers G.D., Trost M.E., Barros C.S.L., Fighera R.A., Irigoyen L.F. \& Silveira I.P. 2012. Lesões do sistema urinário em 1.063 cães. Pesq. Vet. Bras. 32(8):761-771.

Lazaretti P., Kogika M.M., Hagiwara M.K. \& Lustoza M.D. 2006. Concentração sérica de paratormônio intacto em cães com insuficiência renal crônica Arq. Bras. Med. Vet. Zootec. 58(4):489-494.

MacDougall R.C. 2001. Role of uremic toxins in exacerbating anemia in renal failure. Kidney Int. 59(78):S67-S72.

Maxie M.G. \& Newman S.J. 2007. The urinary system, p.425-522. In: Maxie M.G. (Ed.), Jubb, Kennedy, and Palmer's Pathology of Domestic Animals. Vol.2. $5^{\text {th }}$ ed. Saunders Elsevier, St Louis.

Maxie M.G. \& Robinson W.S.F. 2007. Cardiovascular system, p.1-105. In. Maxie M.G. (Ed.), Jubb, Kennedy, and Palmer's Pathology of Domestic Animals. Vol.3. 5th ed. Saunders Elsevier, St Louis.

Newman S.J., Confer A.W. \& Panciera R.J. 2009. Sistema urinário, p.613691. In: McGavin M.D. \& Zachary J.F. (Eds), Bases de Patologia em Veterinária. 4⿳亠丷a ed. Elsevier, Rio de Janeiro.

Peters R.M., Goldstein R.E., Erb H.N. \& Njaa B.L. 2005. Histopathologic features of canine uremic gastropathy: a retrospective study. J. Vet. Intern. Med. 19:315-320.

Polzin D.J. \& Osborne C.A. 1995. Pathothysiology of renal failure and uremia, p.335-367. In: Osborne C.A. \& Finco D.R. (Eds), Canine and Feline Nephrology and Urology. Williams and Wilkins, Baltimore.

Polzin D.J., Osborne C.A., Jacob F. \& Ross S. 2008. Insuficiência renal crônica, p.1721-1751. In: Ettinger S.J. \& Feldman E.C. (Eds), Tratado de Medicina Interna Veterinária: doenças do cão e do gato. Vol.2. 5ª ed. Guanabara Koogan, Rio de Janeiro.

Polzin D., Osborne C. \& O’brient T. 1992. Moléstia dos rins e ureteres, p.2047-2138. In: Ettinger S.J. (Ed.), Tratado de Medicina Interna Veterinária. Vol.4. 3a ed. Manole, São Paulo.

Rackow E.C., Fein I.A., Sprung C. \& Grodman R.S. 1978. Uremic pulmonary edema. Am. J. Med. 64(6):1084-1088.

Thompson K. 2007. Bones and joints, p.1-184. In: Maxie M.G. (Ed.), Jubb, Kennedy, and Palmer's Pathology of Domestic Animals. Vol.1. $5^{\text {th }}$ ed. Saunders Elsevier, St Louis.

Vanholder R., Van Laecke S. \& Glorieux G. 2008. What is new in uremic toxicity? Pediatr. Nephrol. 23:1211-1221. 